\title{
Care Workers and Quality of Care in Nursing Homes in Canada
}

\author{
Zafar Mehdi (PhD), Ramzi Nasser (PhD) \\ ${ }^{1}$ Department of Social Sciences, Dhofar Universty, Salalah, Oman \\ ${ }^{2}$ Education Department, University of Sharjah, Sharjah, UAE
}

\begin{abstract}
The Canadian population is also rapidly ageing and there is a shortage of skilled care workers such as nurses, elder carers and care assistants in Canadian nursing homes. To provide care services for older people in long-term care settings, the advancements in health care knowledge and changing health care environment, make demands on care workers to stay abreast of their knowledge and skills to meet the changing needs of care services in home care settings and residential care facilities. Although there are mandatory and volunteer continuing training programs in long-term care sectors in Canada, the effects of further training of care workers on their career advancement, work situation and quality of care in healthcare and long-term care sector were unclear. The purpose of this chapter is to examine the impact of continuing training of nursing staff in Canadian nursing homes and its association with the quality of care of nursing home residents. Based on theoretical premises of "Lifelong Learning Theory" it is assumed that lifelong learning (further training) of care workers in health care and long-term care is likely to have positive effects on professional development, work situation and quality of patient care. The sample of this study is care workers (nurses, elder carers and care assistants) working in healthcare sector in hospitals, home care settings and long-term nursing homes. This includes care workers; nurses $(n=947)$, elder carers $(n=493)$ and care assistants $(n=1140)$. Data were obtained from the latest Canadian National Survey of Work and Health of Nurses (2005). The study investigated the impact of further training of care workers quality of care in nursing homes in Canada through a multiple regression analysis technique performed on the data. It was found from this study that further training of nurses has significant and positive association with career mobility of the care workers and quality of care of nursing home residents.
\end{abstract}

Keywords: Elderly, Elderly Care Workers, Basic Education, Further Educational Training, Wages, Elderly Wellbeing 